\title{
The impact of language on patient assessed outcomes of specialist consultations
}

\author{
Varsha Kadaba ${ }^{1}$, Kadaba Rajkumar ${ }^{2}$, Daksha Patel ${ }^{3}$ and Ian Murdoch ${ }^{4 *}$ \\ ${ }^{1}$ School of Medicine, Imperial College, London, United Kingdom \\ ${ }^{2}$ Department of Ophthalmology, University Hospital Wales, United Kingdom \\ ${ }^{3}$ Public Health for Eye Care, London School of Hygiene and Tropical Medicine, United Kingdom \\ ${ }^{4}$ Institute of Ophthalmology and Moorfields Eye Hospital NHS Foundation Trust, London, United Kingdom
}

Twenty four percent of the population in Ealing Borough, London is Asian/Asian British compared to the national average of 4\% [1]. Access to interpreters is therefore key for communication amongst the elderly and recent immigrants. An audit in the eye department at Ealing showed that a trained interpreter was present for only 2 out of 80 consultations requiring interpreters [2]. To our knowledge, the advantages between relatives, bilingual health professionals and professional interpreters in ophthalmology consultations have never been compared.

An observational study was conducted over four clinic days. Out of the 6 ophthalmologists conducting clinic consultations, 4 could not speak Hindi/Punjabi and 2 could speak both Hindi and Punjabi. Adult patients who spoke Hindi/Punjabi and required a translator were included in the study after giving written informed consent in their native tongue. Study participants were assigned, using computer randomly generated numbers, to:

a. Doctors speaking Hindi/Punjabi

b. Doctors with a professional interpreter speaking Hindi/Punjabi

c. Doctors not speaking Hindi/Punjabi using the patients' friend/ relative to translate.

Both patients and consulting doctors completed questionnaires to gauge understanding of the consultation. A control group of patients who spoke fluent English and had English consultations were included. Moorfields Ethical Committee granted ethical approval.

A total of 53 consultations were undertaken in the study. Table1 shows numbers in each group and agreement between recorded diagnosis and patients understanding. There was a difference in the understanding of diagnosis between patient and doctor across the groups $(\mathrm{p}=0.058)$, which became significant if viewed as a trend (Chi2

Table 1. Shows numbers in each group and agreement between recorded diagnosis and patients understanding.

\begin{tabular}{|l|c|c|c|}
\hline & $\begin{array}{c}\text { Agreement with } \\
\text { diagnosis }\end{array}$ & $\begin{array}{c}\text { Diagnosis not } \\
\text { understood by } \\
\text { patient }\end{array}$ & $\begin{array}{c}\text { Disagreement with } \\
\text { Diagnosis }\end{array}$ \\
\hline Control & 10 & 0 & 0 \\
\hline Relative & 7 & 6 & 1 \\
\hline Interpreter & 9 & 1 & 0 \\
\hline Bilingual Doctor & 13 & 3 & 0 \\
\hline
\end{tabular}

test for trend $=4.98 \mathrm{p}=0.026$ ) from relative to bilingual to interpreter.

Whilst there was no significant difference in the understanding of diagnosis between patient and doctor across the groups $(\mathrm{p}=0.058)$, and an improving trend $\left(\mathrm{Chi}^{2}\right.$ test for trend=4.98 $\left.\mathrm{p}=0.026\right)$ from relative to bilingual to interpreter. Stronger comprehension was shown concerning drugs and dosage in all groups and similarly with regards to appointments.

On a scale of 1-10 with 10 being complete satisfaction the overall score of satisfaction was the same in all three Asian groups (9.1-9.2) and slightly higher in the control group (9.6). On individual aspects of the consultation (thoroughness, attention, health/eye problems and treatment) patients scored a bilingual doctor higher than an interpreter.

The lowest score on all parameters was a relative translator.

Hospital guidelines in many trusts encourage the use of professional interpreters as it has improved clinical care and broken down the barriers to adequate medical care [3].

However, healthcare professionals still rely on ad hoc interpreters and bilingual staff. Bischoff et al. [4] found that a belief that ad hoc interpreters are 'good enough' existed despite large differences in interpretation quality. Bilingual healthcare workers have been suggested as an alternative. As this study demonstrated, there was improved satisfaction when bilingual doctors held the consultations. An analysis of the role of bilingual healthcare workers by Matthews et al. recognises that bilingual staff are a valuable resource [5].

In conclusion, patients prefer bilingual doctors although their understanding of the consultation is clearer when a professional interpreter is present. Both are preferable to relatives translating. In multi-ethnic societies, bilingual doctors are not practical and interpreters are expensive. Relatives may be an option but clinical staff needs to understand the limitations. Repeating information and visual messaging are essential to support patient comprehension and satisfaction.

Correspondence to: Mr Ian Murdoch, 11-43 Bath Street, London, EC1V 9EL, Tel: 02076086896, Fax: 02076084012; E-mail: I.murdoch@ucl.ac.uk

Key words: specialist consultation, translation, patient understanding, patient satisfaction

Received: October 01, 2015; Accepted: October 23, 2015; Published: October 26,2015 


\section{Competing interests}

The authors declare no conflict of interest.

\section{Funding}

This work was supported by Kings Fund, grant number 2000147.

\section{Contributorship statement}

The original design and concept was by I Murdoch, K Rajkumar, and $\mathrm{D}$ Patel. The analysis was by I Murdoch. The writing up was by $\mathrm{V}$ Kadaba and I Murdoch with review by K Rajkumar and D Patel.

\section{References}

1. Office of National Statistics. Focus on ethnicity and identity, summary report. 2005 . http://www.statistics.gov.uk/hub/people-places/people/identity/index.html (accessed 2 May 2012).

2. Madgulla I, Giddings V, Kamal D, et al. Interpreter services in Moorfields Outreach clinic at Ealing. Moorfields Eye Hospital NHS Healthcare Trust Audit 2010.

3. Leah S Karliner, Elizabeth A Jacobs, Alice Hm Chen, Sunita Mutha (2007) Do professional interpreters improve clinical care for patients with limited english proficiency: A systematic review of the literature. Health Serv Res 42: 727-754. [Crossref]

4. Bischoff A, Hudelson P (2010) Communicating with foreign language-speaking patients: is access to professional interpreters enough? J Travel Med 17:15-20. [Crossref]

5. Matthews C, Johnson M, Noble C, Klinken A (2000) Bilingual health communicators: role delineation issues. Aust Health Rev 23: 104-112. [Crossref]

Copyright: ( 2015 Kadaba V. This is an open-access article distributed under the terms of the Creative Commons Attribution License, which permits unrestricted use, distribution, and reproduction in any medium, provided the original author and source are credited. 